\title{
MULTIPLICATIVE FUNCTIONALS OF A MARKOV PROCESS
}

R. K. GETOOR AND J. W. WOLL, JR.

The present note may best be viewed as an addendum to Meyer's important paper [2]. As such we refer the reader to [2] for all notations, definitions, etc. In particular $\left\{X_{t}\right\}$ will always denote a temporally homogeneous Markov process with state space $X$ (locally compact, separable) satisfying Hunt's hypothesis (A). See [1] or [2].

Let $\left\{M_{t}\right\}$ be a normalized multiplicative functional of $\left\{X_{t}\right\}[2$, p. 136 and p. 141], then Meyer [2, p. 153] has obtained the following very important result: $\left\{M_{t}\right\}$ has the strong Markov property, that is, for each stopping time $T$ and random variable $R \geqq 0$ one has

$$
M_{R+T}(\omega)=M_{T}(\omega) M_{R}\left(\theta_{T} \omega\right)
$$

a.s., $P^{x}$ for each $x$ in $X$. Unfortunately Meyer's proof contains a slight gap (in the proof of Theorem 4.2 on p. 152 of [2]) and the result is not valid without additional assumptions on $\left\{M_{t}\right\}$ as the following example shows. Let $\left\{X_{t}\right\}$ be one-dimensional Brownian motion (so that $X$ is the real line) and define $M_{t}(\omega)=1$ for all $t \geqq 0$ if $X_{0}(\omega) \neq 0$ and $M_{t}(\omega)=0$ for all $t \geqq 0$ if $X_{0}(\omega)=0$. It is easy to see that this defines a multiplicative functional of $\left\{X_{t}\right\}$ since $P^{x}\left(X_{t}=0\right)=0$ for all $t>0$ and $x$ in $X$. On the other hand if $T$ is the first passage time to 0 it is immediate that (1.1) is not valid.

We state the following criterion for the strong Markov property.

THEOREM. The normalized multiplicative functional $\left\{M_{t}\right\}$ has the strong Markov property if and only if for every stopping time $T$ and $x$ in $X$,

$$
P^{x}\left[X_{T} \in N, M_{T}>0\right]=0,
$$

where $N$ is the (universally measurable) set of nonpermanent points of $\left\{M_{t}\right\}$, i.e., $N=\left\{x: P^{x}\left(M_{0}=0\right)=1\right\}$.

Proof. The sufficiency may be established exactly as in [2], the condition (1.2) being just the condition necessary to make the proof of Theorem 4.2 [2, p. 152] valid. (One can give a much simpler proof of the sufficiency using resolvents instead of semi-groups.) To prove the necessity let $R=\inf \left\{t: M_{t}=0\right\}$, then $R$ is a stopping time and the right continuity of $\left\{M_{t}\right\}$ implies that $P^{x}\left(M_{R}>0, R<\infty\right)=0$ for all $x$. Let $T$ be any stopping time and consider the random variable

Received by the editors May 31, 1962 and, in revised form, November 19, 1962. 
$H(\omega)=T(\omega)+R\left(\theta_{T} \omega\right)$. If $x$ is fixed the strong Markov property yields

$$
E^{x}\left[M_{H}, H<\infty\right] \leqq E^{x}\left[M_{T} E^{X(T)}\left(M_{R}, R<\infty\right)\right]=0 .
$$

Thus $H=T+R\left(\theta_{T}\right) \geqq R$ on $\{H<\infty\}$ and so $T+R\left(\theta_{T}\right) \geqq R$, both statements holding a.s., $P^{x}$. Therefore

$$
\begin{aligned}
P^{x}\left[X_{T} \in N, M_{T}>0\right] & =P^{x}\left[X_{T} \in N, T<R\right] \\
& \leqq E^{x}\left[P^{X(T)}(R>0), X_{T} \in N\right] \\
& =0,
\end{aligned}
$$

since $P^{y}(R>0)=0$ for all $y$ in $N$.

If $T=\inf \left\{t>0, X_{t} \in N\right\}$ is a stopping time, then (1.2) can be replaced by $P^{x}\left(M_{T}>0, T<\infty\right)=0$ for all $x$. This is the case if $N$ is nearly analytic. The multiplicative functional defined in the second paragraph gives an example of a nonperfect multiplicative functional since any perfect multiplicative functional has the strong Markov property. (See $[2$, p. 136] for the definition of a perfect multiplicative functional.) As an example of a strongly Markov multiplicative functional for which the corresponding semi-group $\left\{Q_{t}\right\}$ is not exactly subordinate to $\left\{P_{t}\right\}$ (the semi-group of $\left\{X_{t}\right\}$ ), let $\left\{X_{t}\right\}$ be translation to right along the real line at unit speed. Put $M_{t}(\omega)=0$ if $t+X_{0}(\omega) \geqq 0$ and $X_{0}(\omega) \leqq 0$, and $M_{t}(\omega)=1$ otherwise. It is easily verified that this example has the desired properties.

\section{REFERENCES}

1. G. A. Hunt, Markoff processes and potentials. I, Illinois J. Math. 1 (1957), 44-93.

2. P. A. Meyer, Fonctionelles multiplicatives et additives du Markov, Ann. Inst. Fourier (Grenoble) 12 (1962), 125-230.

UNIVERSITY OF WASHINGTON 